\title{
Retraction: Controlling Web Services and 802.11 Mesh Networks
}

\author{
Chen-shin Chien ${ }^{1}$ and Jason Chien ${ }^{2}$ \\ ${ }^{1}$ Department of Industrial Education, \\ National Taiwan Normal University, Taipei County, Taiwan \\ chiendoc@ntnu.edu.tw \\ ${ }^{2}$ China Unversity of Science and Technology Computing Center \\ China Unversity of Science and Technology, Taipei County, Taiwan \\ jason034@cc. cust. edu. tw
}

Several conference proceedings have been infiltrated by fake submissions generated by the SCIgen computer program. Due to the fictional content the chapter "Controlling Web Services and 802.11 Mesh Networks" by "Chen-shin Chien and Jason Chien" has been retracted by the publisher. Measures are being taken to avoid similar breaches in the future. 